\title{
gु \\ Diffusion jumps of single atoms into vacancies in an iron monolayer studied by nuclear resonant scattering
}

\author{
G. Vogl* and E. Partyka-Jankowska \\ Faculty of Physics, University of Vienna, Strudlhofgasse 4, A-1090 Vienna, Austria \\ M. Zając \\ Faculty of Physics and Applied Computer Science, AGH University of Science and Technology, Al. Mickiewicza 30, \\ 30-059 Cracow, Poland \\ and European Synchrotron Radiation Facility, 6 rue Jules Horowitz, BP 220, 38043 Grenoble Cedex, France
}

A. I. Chumakov

European Synchrotron Radiation Facility, 6 rue Jules Horowitz, BP 220, 38043 Grenoble Cedex, France

(Received 27 May 2009; published 4 September 2009)

\begin{abstract}
We study the structure of a model surface, i.e., an Fe monolayer on W(110) by nuclear resonant scattering of $\mathrm{x}$ rays. We conclude that at room temperature the structure is nearly perfect, whereas when increasing the temperature up to $770 \mathrm{~K}$ an increasing fraction of the Fe atoms feels the appearance of defects manifested by a beat structure due to an electric field gradient. The field gradient is caused by an asymmetry of the neighborhood of part of the atoms and attributable to an increasing fraction of vacancies in the monolayer. With increasing temperature the beat structure gets smeared due to the onset of dynamics in the surface, i.e., the motion of the defects and consequently of the atoms. From the temperature dependence of the amplitude of the beat we deduce the vacancy concentration, thus the vacancy formation energy $[0.17(5) \mathrm{eV}]$ and from the broadening with less accuracy we deduce the vacancy migration energy $[0.16(6) \mathrm{eV}]$ resulting in $0.33(11) \mathrm{eV}$ for the activation energy of diffusion.
\end{abstract}

DOI: $10.1103 /$ PhysRevB.80.115406

PACS number(s): 68.35.Fx, 61.72.Dd, 76.80.+y, 66.30.-h

\section{INTRODUCTION}

We report on defect formation and motion in an $\mathrm{Fe} /$ $\mathrm{W}(110)$ surface monolayer (ML). The motion of atoms in a monolayer is more difficult to follow than atom motion in the bulk. Whereas information on defect motion in the bulk has been the issue of a great number of papers (see, e.g., Ref. 1) only very few investigations have attempted to study the two-dimensional system of a surface because of the difficulties (a) to produce a clean surface and (b) to select methods which can study the motion of single surface atoms.

Until now most experimental information about diffusion in a surface stems from field ion microscopy. ${ }^{2-4}$ These studies were limited to adatoms: the hopping of adatoms on the surface or the exchange of adatoms (e.g., Pt, W, Re) with surface atoms on (110) and (100) surface planes of Pt, Ir, Ni, and $\mathrm{W}$ have been followed. By this way the activation energy for surface diffusion of single metal atoms of a number of elements on $\mathrm{W}(110)$ has been conjectured. Values range from $E_{d}=0.5 \mathrm{eV}$ for $\mathrm{Ni}$ and $\mathrm{Pd}$ atoms to $1.0 \mathrm{eV}$ for Re atoms. ${ }^{2,3,5,6}$ Values for $\mathrm{Fe}$ atom diffusion were not given, and the motion of possible vacancies involved in the process cannot be followed directly, nor can the formation or migration energies be determined separately. Existing extensive local densityfunctional calculations ${ }^{7}$ of formation and migration energies of self-atoms can therefore only partially be compared with experimental reality.

For studying diffusion by way of scanning tunneling microscopy (STM) the interaction of the STM tip with the surface atoms can be exploited for manipulating the surface, but at the same time strongly influences the diffusion process. ${ }^{8}$
To investigate metal surfaces and in particular Fe surfaces by STM is difficult because of insufficient corrugation of the local density of states. Vacancy motion in a semiconductor surface has been observed by means of STM on the $\mathrm{Ge}(111) c(2 \times 8)$ surface. ${ }^{9}$ However again the vacancy (or a pair of vacancies) has been created artificially by extracting a selected Ge atom with the STM tip.

What is so difficult with STM for self-atoms and vacancies in a surface has been achieved since 20 years for adatoms on surfaces. A multitude of investigations of adatoms and molecules on surfaces has been successfully performed by STM (e.g., Ref. 10 and references therein). In a number of investigations it has even been possible to follow the motion of adatoms using fast STM taking between 10 and 100 pictures per second. ${ }^{11,12}$

Studies of the dynamics of pure surfaces without adatoms and without intervention by the tip of the STM have only recently been pioneered. Using the device constructed for the present investigations ${ }^{13}$ and the method of incoherent nuclear inelastic scattering of synchrotron radiation in grazing incidence geometry, the vibrational density of states in the uppermost layers of an Fe on W(110) film was determined. ${ }^{14}$ It was possible not only to separate the dominating contribution of the surface monolayer but also to deduce the contributions from the next layers. In order to receive full information on the dynamics in a thin film it is of interest to complement the information on collective dynamics by determining the motion of single atoms.

In the present paper the diffusion in a monolayer of a pure metal has been experimentally tackled and successfully accomplished by a method which does not make use of a mi- 
croscope tip and consequently is free of potential artifacts and works on the time scale of the diffusion jumps. The method of our choice was nuclear resonant scattering (NRS) of synchrotron radiation. Another scattering method exploited successfully in the past for studying surface diffusion, i.e., quasielastic Helium atom scattering, ${ }^{15}$ has a time resolution about a factor thousand weaker than the resolution of NRS. The scattering methods work "in reciprocal space" and full information is gained by Fourier transformation. Their results profit from the ample statistics on which they rely because they are averages of a number of events of the same type. For reviews on scattering methods for studying the motion of single atoms see Refs. 16 and 17.

We have chosen an uncoated Fe monolayer grown on $\mathrm{W}(110)$ mainly because this system is probably the best investigated one among metallic surfaces. The high melting temperature of $\mathrm{W}$ and therefore low diffusivity of other metal atoms into $\mathrm{W}$ at moderate temperatures motivated the frequent use of $\mathrm{W}$ for growing thin metallic films on $\mathrm{W}$ surfaces, not only on (110) surface planes ${ }^{14,18-23}$ but also on (100) planes. ${ }^{24}$ Thin Fe films on W(110) (and also on $\mathrm{W}(100))$ feel a large tensile strain, since the lattice constant of $\mathrm{Fe}$ is 9.4 percent lower than that of $\mathrm{W}$. Therefore an iron monolayer on $\mathrm{W}(110)$ is a model system for pseudomorphic growth, i.e., the $\mathrm{Fe}$ atoms are aligned in registry with the $\mathrm{W}$ atoms and is stable up to $1200 \mathrm{~K}$ as known from Auger electron spectroscopy (AES). ${ }^{24}$ Investigations up to $900 \mathrm{~K}$ should be feasible without risking the change of the structure. 1 and $2 \mathrm{ML}$ flat Fe films on a W(110) substrate are ferromagnetic at $0 \mathrm{~K},{ }^{25}$ but paramagnetic ("nonmagnetic") at and above room temperature with a Curie temperature for the pure $1 \mathrm{ML}$ film of $210 \mathrm{~K}$ as determined by conversion electron Mössbauer spectroscopy ${ }^{26,27}$ (CEMS) or $225 \mathrm{~K}$ as determined by spin-polarized electron diffraction. ${ }^{28} \mathrm{Fe}$ films on $\mathrm{W}$ met interest starting from the early 1980s but very little consolidated information on diffusion in and on these films is available except from the work in Kirschner's group. ${ }^{22}$ There, from the evolution of the concentration profile around an Fe dot evaporated on W(110), its extension reaching about $25 \mu \mathrm{m}$ after $24 \mathrm{~min}$ at $820 \mathrm{~K}$, the diffusion coefficient of a "top $\mathrm{Fe}$ atom" on an Fe monolayer was estimated as about $10^{-13} \mathrm{~m}^{2} \mathrm{~s}^{-1}$.

\section{EXPERIMENT}

\section{A. Idea of nuclear resonant scattering of synchrotron radiation}

Our idea was to follow the motion of defects in an ${ }^{57} \mathrm{Fe}$ monolayer by taking advantage of the sensitivity of recoilless nuclear resonance. We made use of the synchrotron variant of recoilless nuclear resonance absorption, the Mössbauer effect, but in time domain instead of energy domain, denominated NRS. We aimed at using at the same time the Mössbauer effect's sensitivity to fluctuations of the hyperfine fields in the probe's neighborhood and the diffusionally accelerated decay of the intensity re-emitted after nuclear resonance.

Studying diffusion in a monolayer using the conventional Mössbauer effect is nearly hopeless due to absolutely insuf-
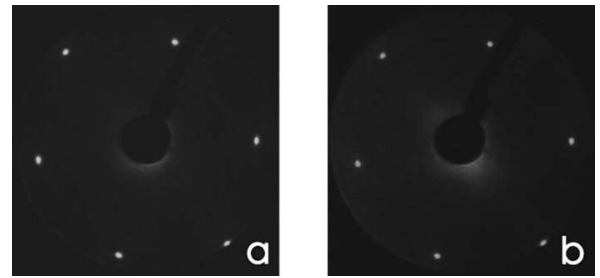

FIG. 1. LEED pattern of an iron monolayer before (a) and after (b) the NRS measurements obtained at a beam energy of $62 \mathrm{eV}$.

ficient intensity of a conventional Mössbauer source even with a forbidding source strength of one Curie. The x-ray beam from third generation synchrotrons with their high brilliance, on the contrary, has sufficient spectral density even for the excitation of nuclear levels with narrow bandwith $\left(4.7 \mathrm{neV}\right.$ for $\left.{ }^{57} \mathrm{Fe}\right)$. In the case of thin films one has to resort to grazing incidence in order to increase the "effective" thickness that leads to an increased delayed intensity. ${ }^{29}$

\section{B. Sample preparation and characterization}

The experiment was carried out at the nuclear resonance beamline ID18 (Ref. 30) at the European Synchrotron Radiation Facility using a recently constructed multifunctional ultrahigh vacuum (UHV) system. ${ }^{13}$ The system consists of a preparation chamber equipped with standard surface preparation and characterization devices and a chamber for $\mathrm{x}$-ray scattering measurements in grazing incidence geometry, all equipment placed directly at the synchrotron beam in order to avoid transportation which risks breaking the UHV and deteriorating the clean surface.

The W(110) substrate was cleaned in the typical manner by several annealing treatments at $1600 \mathrm{~K}$ under oxygen pressure of $10^{-7}$ mbar followed by flashing at $2100 \mathrm{~K}$. The cleanliness of the substrate was checked by carbon/tungsten AES signals and by low-energy electron diffraction (LEED). For a clean $\mathrm{W}$ surface a perfect $(1 \times 1)$ pattern has been registered. The misfit between the $\mathrm{W}$ and Fe lattice constants results in a strained $\mathrm{Fe}$ monolayer being completed at the coverage of 0.82 monolayer of bulk $\mathrm{Fe}(110)$, i.e., at the thickness of $1.66 \AA$. Using an electron beam evaporator equipped with flux monitor and calibrated with a quartzbalance monitor a layer of $1.6 \AA$ of the iron isotope ${ }^{57} \mathrm{Fe}$ (concentration of ${ }^{57} \mathrm{Fe} 95 \%$ ) was deposited with a rate of $1 \AA \mathrm{min}^{-1}$ at $770 \mathrm{~K}$. The pressure during film growth was below $2 \times 10^{-10}$ mbar and the thickness precision of the deposited layer was about $0.1 \AA \mathrm{Fe}$. We aimed at obtaining a flat Fe monolayer. A $(1 \times 1)$ LEED pattern registered after deposition (Fig. 1(a)) confirmed a pseudomorphic growth of the monolayer. After preparation, the specimen was cooled down by switching off the heating, and a series of NRS measurements at increasing temperatures starting from room temperature was performed.

\section{Measurement}

For NRS we used synchrotron radiation monochromized to an energy bandwith of $0.75 \mathrm{meV}$ at $14.413 \mathrm{keV}$ and vertically focused by $\mathrm{KB}$ optics ${ }^{31}$ to $7 \mu \mathrm{m}$. All spectra were 


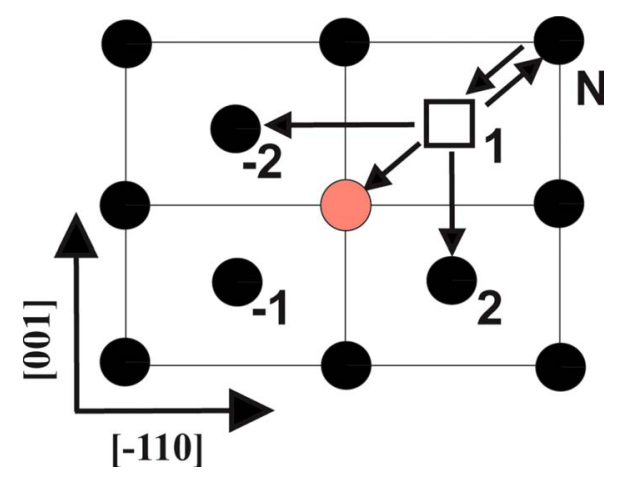

FIG. 2. (Color online) Diffusion of Fe atoms in an (110) surface plane. $1,-1,2$, and -2 designate possible vacancy positions at nearest-neighbor $(\mathrm{NN})$ sites of the probe atom, $\mathrm{N}$ a site outside the NN shell. Vacancy jumps between positions 1 and 2 and between 1 and -2 are effective jumps each composed of two $\mathrm{NN}$ jumps with jump frequency $w$.

taken in grazing incidence geometry at the angle of $5 \mathrm{mrad}$ that corresponds to the critical angle of the Fe/W system. ${ }^{32}$ The direction of the synchrotron beam was parallel to the [110] direction of the monolayer if neglecting the small incidence angle.

The average time for collecting a spectrum depended on the temperature. At room temperature, a period of $20 \mathrm{~min}$ was enough to get a spectrum with sufficient statistics, whereas at $770 \mathrm{~K}$ at least a one hour measurement was necessary for receiving a spectrum of equal quality. Due to the high temperatures, the adsorption of residual gases (mainly $\mathrm{CO}, \mathrm{H}_{2} \mathrm{O}$, and $\mathrm{CO}_{2}$ ) was negligible as can be seen in the LEED pattern taken after the completed NRS measurements (Fig. 1(b)). The pattern does not show presence of $p(2 \times 2)$ or mixture of $p(2 \times 2)$ and $p(3 \times 2)$ domains that were observed in an $\mathrm{Fe}$ monolayer after exposure to residual gases at room temperature. ${ }^{33}$

\section{SHORT SUMMARY OF THEORY OF DIFFUSION STUDIES BY NUCLEAR RESONANT SCATTERING OF SYNCHROTRON RADIATION}

As has been demonstrated in the past, interference of highly brilliant, polarized and pulsed synchrotron radiation can probe jumps of single atoms directly in time domain in the range of nanoseconds and on the space scale of Angstroms (see e.g., Refs. 34 and 35). A highly monochromatic synchrotron $\mathrm{x}$-ray pulse excites the nuclear transition from the ground state ( $\operatorname{spin} I=1 / 2$ ) of the ${ }^{57} \mathrm{Fe}$ nucleus to the first excited nuclear state at $14.413 \mathrm{keV}(I=3 / 2)$. The ensemble of excited ${ }^{57} \mathrm{Fe}$ nuclei re-emits the radiation in phase with a time delay (lifetime of the excited state $141 \mathrm{~ns}$ ). This is called "delayed intensity" to distinguish from the prompt intensity of the primary synchrotron beam.

Here we only sketch the theory, an outline of the theory being given in Ref. 36 together with some information what has been achieved so far in the field of diffusion. In that work for sake of simplicity the vacancy concentration had been kept constant different from the realistic case we deal with here. The effect of the atoms changing sites by way of

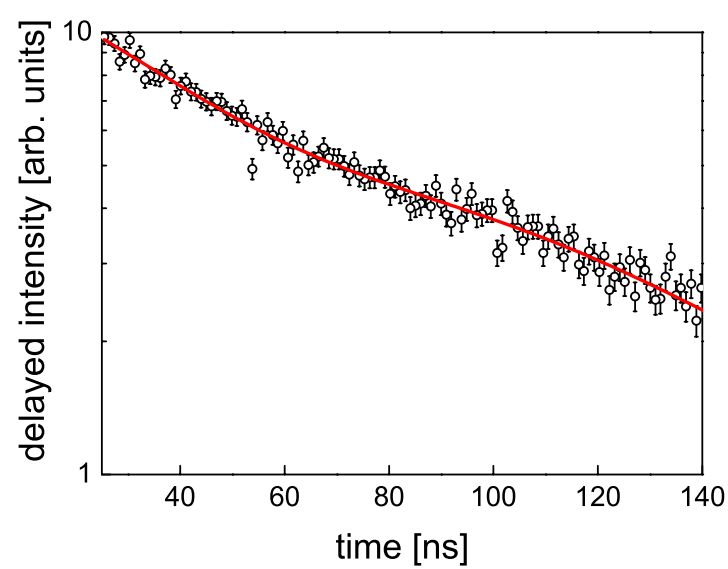

FIG. 3. (Color online) Delayed intensity from nuclear resonant scattering at an as-prepared Fe monolayer measured during $20 \mathrm{~min}$ at room temperature. The smooth line is a fit which is discussed in the text.

vacancies in absence of fluctuating hyperfine interactions is described by a "jump frequency matrix." The matrix elements are the vacancy jump rates for leaving a site or changing from one site $1,-1,2,-2$, or $\mathrm{N}$ (compare Fig. 2) to another site. For self-diffusion, i.e., diffusion of just one type of atoms in the absence of foreign atoms, all frequencies are identical. This vacancy jump may or may not lead to a jump of the probe atom itself. Jumps of the probe atom produce a diffusionally accelerated decay of the NRS intensity.

The electric field gradient tensor acting at the nucleus of a diffusing atom reorients when a vacancy jumps in the nearest neighborhood of the probe atom containing the nucleus. The jumps bring about relaxations in the quadrupole interactions and consequently the ensemble of nuclei loses its phase relation. This yields again information on the jump frequency.

Diffusionally accelerated decay and quadrupole interactions come into play then and only then when a vacancy approaches the probe atom. For sake of simplicity this is assumed to happen only if the vacancy enters into the nearest neighbor shell of the probe atom. The interaction therefore is weighted by a probability factor into which enter the vacancy concentration and the vacancy jump frequency.

Due to the presence of an electric field gradient (EFG) in the Fe monolayer, the excited ${ }^{57} \mathrm{Fe}$ state splits into two substates with magnetic quantum numbers $m_{e}= \pm 1 / 2$ and $\pm 3 / 2$ (neglecting magnetic interactions). The ground state remains degenerate with $m_{g}= \pm 1 / 2$. The interference of two emitted waves corresponding to a de-excitation of both excited sublevels, results in a so-called quantum beat pattern in the time domain, ${ }^{37,38}$ which depends on the magnitude of the EFG tensor and the orientation of its main axis relative to the synchrotron beam (for conventional Mössbauer effect, registered in energy domain, the spectrum splits into different resonance lines). The components of the EFG tensor are the gradients of the electric field in different directions due to the electric anisotropy, be it the anisotropy inherent in the surface or the anisotropy due to neighboring vacancies. The beat frequency is larger the higher the quadrupole interaction acting on the nucleus. Fluctuation of the quadrupole interaction due to diffusion causes an increasing smearing of the beats, 


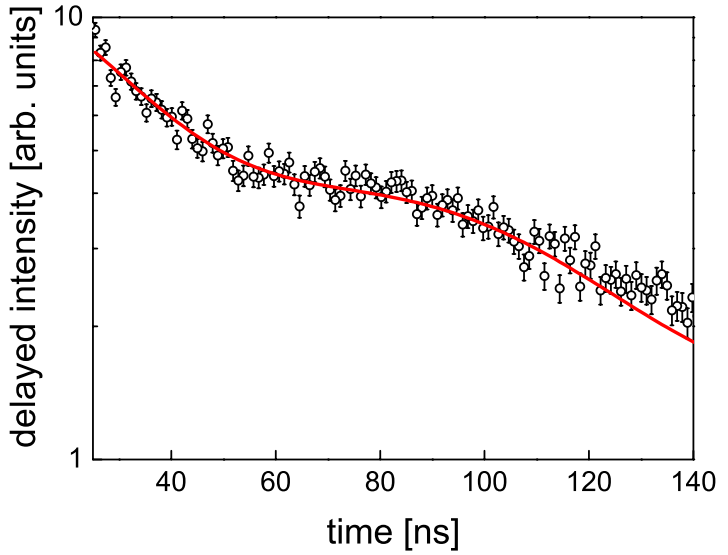

FIG. 4. (Color online) Delayed intensity from nuclear resonant scattering at an Fe monolayer, measured during one hour at $T$ $=570 \mathrm{~K}$. The smooth line is a fit.

the faster the vacancies in the neighborhood and the ${ }^{57} \mathrm{Fe}$ atom itself move. For mobile probe atoms the decay of the NRS intensity in time continues to steepen with increasing jump frequency.

Owing to the polarization dependence of NRS, there is one special case, when a time spectrum without beats appears for the case of a purely electric hyperfine interaction. ${ }^{36,37}$ This is the case when the EFG main axis is perpendicular to the polarization vector and to the wave vector of the incoming synchrotron radiation, which for grazing incidence happens when the EFG main axis is perpendicular to the surface (the plane of the monolayer). In this particular case, only the transition with the selection rule $\Delta m=0$ can be excited. This leads to a single transition and therefore no quantum beat in the NRS spectrum is observed. Any other direction of the main EFG axis results in the emergence of a beat pattern in the NRS spectrum, i.e., a beat appears as soon as through the action of an additional field gradient, e.g., from a defect, the resultant field gradient is not any longer vertical to the surface.

Finally it has to be mentioned that the coherent and collective nature of NRS of synchrotron radiation leads to a speedup compared to that of an isolated nucleus. This effect is especially strong in grazing incidence reflection geometry because the interference of the multiply reflected waves enhances the decay. Dynamic theory for grazing incidence at the Fe monolayer on W yielded an apparent decay time of 88 ns and this value was taken for fitting the spectra.

\section{EXPERIMENTAL RESULTS}

Figure 3 shows the time dependence of the re-emitted intensity ("delayed intensity") from resonance at the 14.413 $\mathrm{keV}$ nuclear level registered at room temperature. For all spectra evaluation starts at $25 \mathrm{~ns}$ because of an artifact at 23 ns coming from the detector. Several characteristics deserve attention. The re-emitted intensity decays essentially exponentially in time (small deviations will be discussed later). This implies that there is essentially no additional electric field gradient beyond the electric field gradient from the an-

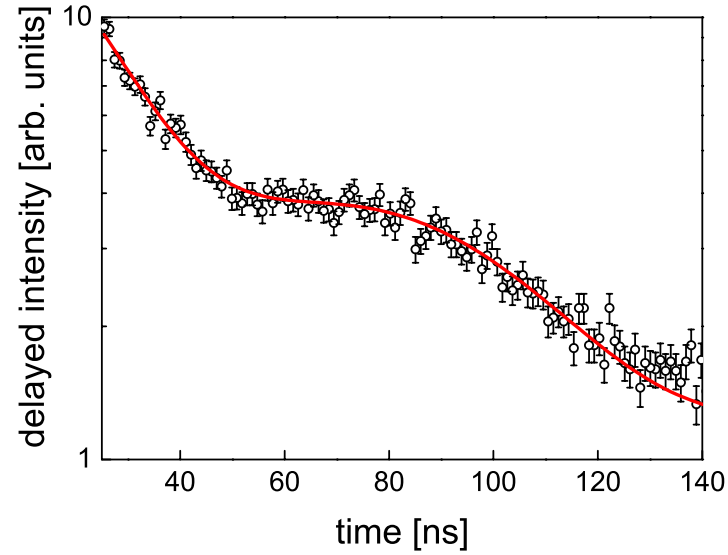

FIG. 5. (Color online) Delayed intensity from nuclear resonant scattering at an Fe monolayer measured during one hour at $T$ $=670 \mathrm{~K}$. The smooth line is a fit.

isotropy inherent in the surface, i.e., the surface is practically perfect and undisturbed. Already at a measuring temperature of $570 \mathrm{~K}$ indication for an incipient beat, i.e., a deviation from the exponential decay, appears (Fig. 4), with its minimum around $45 \mathrm{~ns}$. With stepwise increasing temperature, the beat structure becomes more and more distinct (Figs. 5 and 6) and is strongest at $870 \mathrm{~K}$ (Fig. 7), the highest temperature which was attainable in order not to risk Fe diffusion into the $\mathrm{W}$ substrate and thereby to destroy the $\mathrm{Fe}$ monolayer.

When the temperature is finally lowered again to room temperature, the beat structure disappears and the original spectrum is recovered, proving that thermally activated defects are responsible for the beat. The recovery, though, is not perfect after the measurement at $770 \mathrm{~K}$ (see Fig. 10) and even less so after the measurement at $870 \mathrm{~K}$.

Best fit of the NRS spectra with the theory developed in Ref. 36 was received with a combination of quadrupole interaction energy $Q_{N}(N$ indicates "normal to the surface") caused by the anisotropy of the surface of $21(2) \mathrm{neV}$ and a fluctuating quadrupole interaction $Q_{D}(D$ indicates "defect") caused by inhomogeneities, i.e., lattice defects, which de-

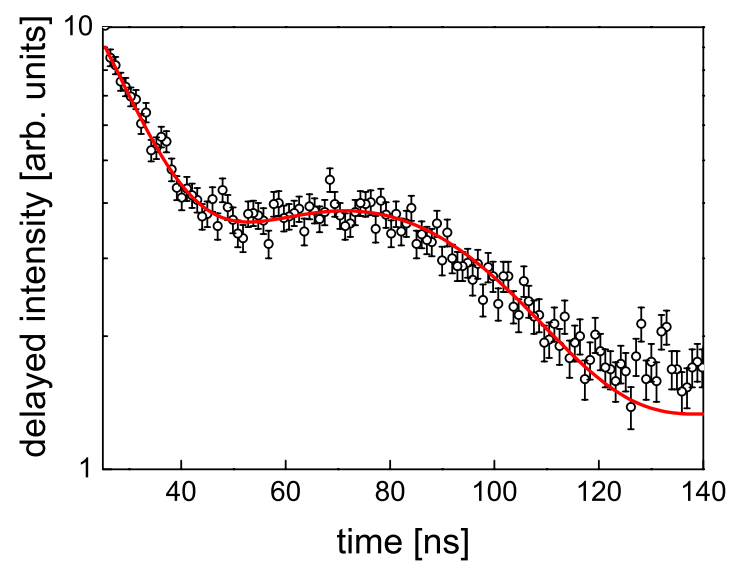

FIG. 6. (Color online) Delayed intensity from nuclear resonant scattering at an Fe monolayer measured during $1.5 \mathrm{~h}$ at $T=770 \mathrm{~K}$. The smooth line is a fit. 


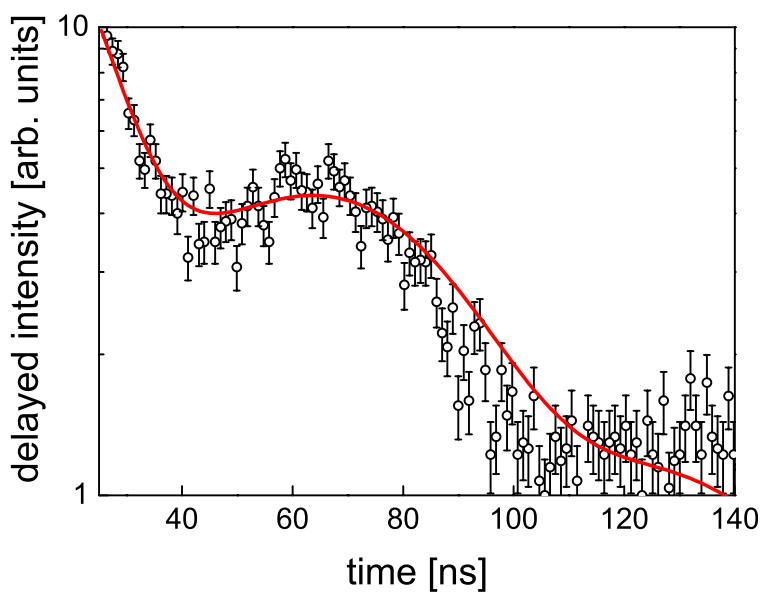

FIG. 7. (Color online) Delayed intensity from nuclear resonant scattering at an Fe monolayer measured during $1.5 \mathrm{~h}$ at $T=870 \mathrm{~K}$. The fit with the theory developed in Ref. 36 is not optimal probably because of irreversible structural changes occurring at $T=870 \mathrm{~K}$ (see Sec. V D 3).

pends on temperature and varies from $70(5) \mathrm{neV}$ at $570 \mathrm{~K}$ to $87(10) \mathrm{neV}$ at $770 \mathrm{~K}$ and an estimated $104(15) \mathrm{neV}$ at $870 \mathrm{~K}$. The quadrupole interaction $Q_{N}$ of $21 \mathrm{neV}$ caused by the anisotropy of the surface is about $30 \%$ lower than Przybylski's ${ }^{39}$ value (34 neV) which in default of any other information available at that time has been used in Ref. 36 .

From the experimental results of Ref. 33 we know that this value must have been caused by gaseous adatoms since in Przybylski's CEMS studies ${ }^{39}$ lasting for many days although under UHV conditions $\left(3 \times 10^{-10}\right.$ mbar $)$ definitely a layer of gas adatoms must have grown. Przybylski's value is not very different from what Partyka ${ }^{33}$ has found for an iron monolayer upon adsorption of residual gases in an UHV system.

The quadrupole interaction $Q_{D}$ from the defects arises from the perturbation of the probe atom on arrival of a defect in the probe atom's neighborhood. The quadrupole interaction $Q_{D}$ then enters into a joint Hamiltonian with the quadrupole interaction $Q_{N}$ caused by the anisotropy of the surface. As explained in Sec. III, because of the in-plane polarization of the synchrotron beam, $Q_{N}$ will produce no quantum beat as long as the main axis of the surface quadrupole tensor is normal to the surface, but is "set into action" when a defect arrives in the probe atom's neighbor shell(s). Then the surface quadrupole interaction adds up in energy to the quadrupole interaction from the anisotropy caused by the defect's perturbation of the symmetry. From the resulting quadrupole interaction, i.e., from the amplitude of the beat, the concentration of defects can be deduced.

With increasing temperature the width of the beat with its minimum at about $45 \mathrm{~ns}$ and its maximum at about $80 \mathrm{~ns}$ becomes larger (is smeared out) due to the thermal fluctuation of the defect structure which brings about a relaxation of the quadrupole interaction. Furthermore, the intensity decay steepens. From the temperature dependence the velocity of the defect motion can be deduced. This yields the jump frequency of the defects and its temperature dependence.
TABLE I. Concentration $c$ and jump frequency $w$ of vacancies and diffusion coefficient $D$ as derived from the plots of the delayed nuclear resonant scattering intensity (Figs. 3-7). At $300 \mathrm{~K}$ the concentration of defects is too small to determine the jump frequency.

\begin{tabular}{cccc}
\hline \hline$T$ & $c$ & $w$ & $D$ \\
$(\mathrm{~K})$ & $(\%)$ & $(\mathrm{MHz})$ & $\left(10^{-12} \mathrm{~m}^{2} \mathrm{~s}^{-1}\right)$ \\
\hline 300 & $<3.0$ & & \\
570 & $4.0 \pm 1.0$ & $0.5 \pm 0.3$ & $0.15 \pm 0.13$ \\
670 & $8.5 \pm 1.5$ & $1.9 \pm 0.3$ & $1.20 \pm 0.40$ \\
770 & $11.0 \pm 1.0$ & $1.8 \pm 0.2$ & $1.50 \pm 0.30$ \\
870 & $14.0 \pm 2.0$ & $3.2 \pm 0.4$ & $3.40 \pm 1.20$ \\
\hline
\end{tabular}

\section{DEFECT TYPE, CONCENTRATION, MOTION, AND DIFFUSION}

Thermal production on heating and disappearance on recooling suggest that the thermally activated defects in the $\mathrm{Fe} / \mathrm{W}(110)$ monolayer are vacancies. This is compatible with the calculations of Spisak and Hafner ${ }^{7}$ that vacancies in a nonmagnetic $1 \mathrm{ML} \mathrm{Fe} / \mathrm{W}(110)$ are stable and highly mobile.

\section{A. Vacancy concentration}

As has been argued in Sec. IV the size of the quadrupole interaction caused by the vacancy scales with the probability that a vacancy arrives in the neighborhood of the probe atom. In our model we restrict the vacancy's influence to its arrival in the nearest-neighbor shell in order to end up with a sufficiently simple description. The concentrations derived via fits with the theory (Sec. III and Ref. 36) are given in Table I for temperatures between 300 and $870 \mathrm{~K}$. The Arrhenius plot (Fig. 8) for vacancy formation yields a formation energy $E_{f}$ of $0.17(5) \mathrm{eV}$. In our earlier simulations ${ }^{36}$ for sake of simplicity we have kept the defect concentration constant. The present experiments indicate that in a pure monolayer (no foreign adatoms) a substantial fraction of vacancies is

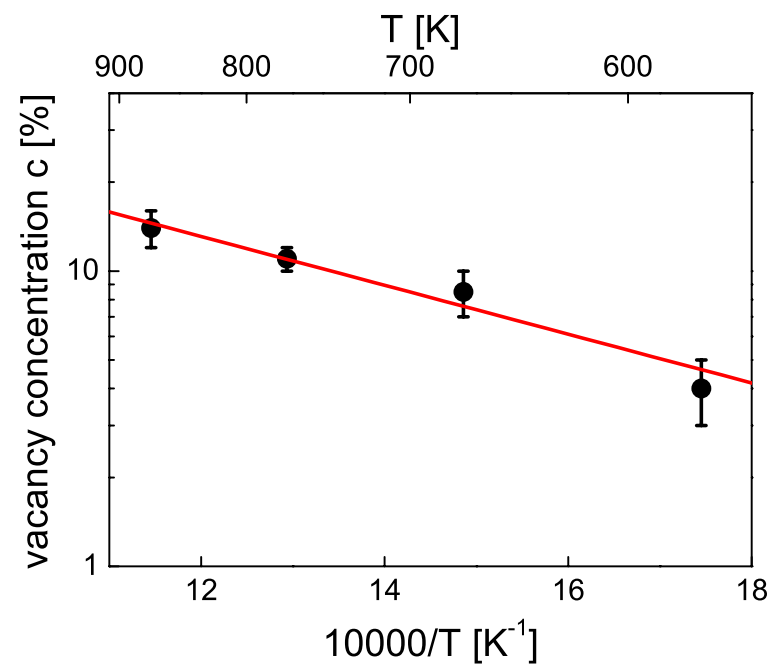

FIG. 8. (Color online) Vacancy concentration $c$ as function of temperature in semilogarithmic Arrhenius plot. 


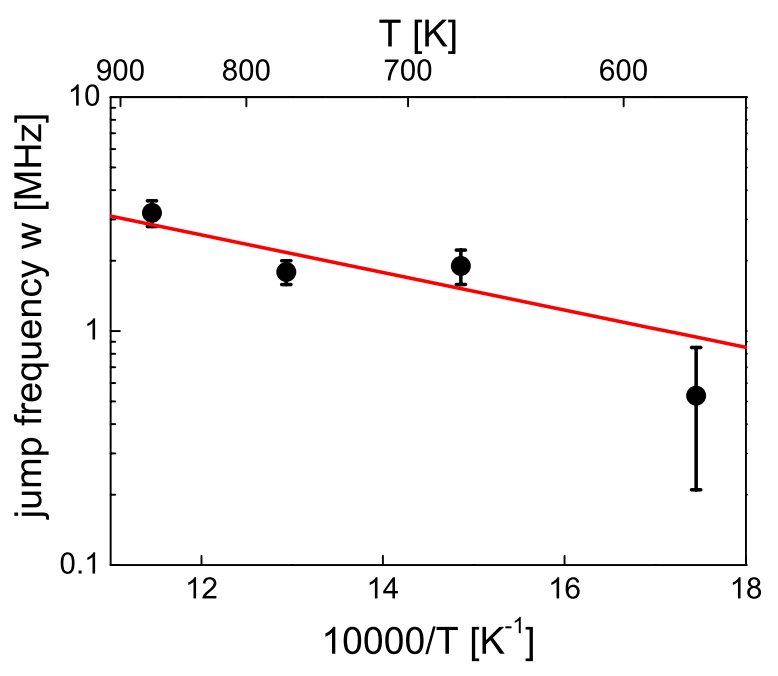

FIG. 9. (Color online) Vacancy jump frequency $w$ as function of temperature in semilogarithmic Arrhenius plot.

formed at elevated temperatures. From their calculations Spisak and Hafner ${ }^{7}$ received a value $E_{f}=0.10 \mathrm{eV}$ for the formation energy of vacancies in a nonmagnetic Fe monolayer on $\mathrm{W}(110)$. This is not too different from the value determined from our experiments $E_{f}=0.17(5) \mathrm{eV}$. Spisak and Hafner note that their calculations are based on $4 \%$ vacancies rather than a single vacancy, a problem which we face in our simulations, too (see below). They add, however, that they do not expect any significant deviations from the calculated energy barriers due to vacancy-vacancy correlations present in their models. The question remains where the atoms released from the vacancies go. There is a considerable tendency for dislocations to form even in a nearly perfect monolayer. ${ }^{40}$ We suggest that the atoms released from vacancies collect in form of dislocations. Alternatively, from Spisak and Hafner's work ${ }^{7}$ one might infer that the atoms released from the vacancies might end up in dimers, i.e., configurations of two atoms occupying together just one site in the monolayer slightly (by $0.19 \AA$ ) protruding from the surface.

\section{B. Vacancy motion}

The vacancy migration energy is less well determinable than the formation energy. A fit of the temperature dependence of the jump frequency (Fig. 9) derived from fitting the beat smearing (Table I) yields $E_{m}=0.16(6) \mathrm{eV}$, but we have to be aware of the large uncertainty in fitting the smearing of the beat. Spisak's and Hafner's ${ }^{7}$ arguments for the vacancy migration energy are sophisticated: they propose three different possible vacancy jumps in Fe/W(110) (their Fig. 9) and receive for the lowest rate determining barrier $0.6 \mathrm{eV}$, about four times the value of $0.16 \mathrm{eV}$ which we deduce from our data.

\section{Diffusion coefficient}

Having suggested that the defects being formed at increasing temperatures in $\mathrm{Fe} / \mathrm{W}(110)$ are vacancies we have to

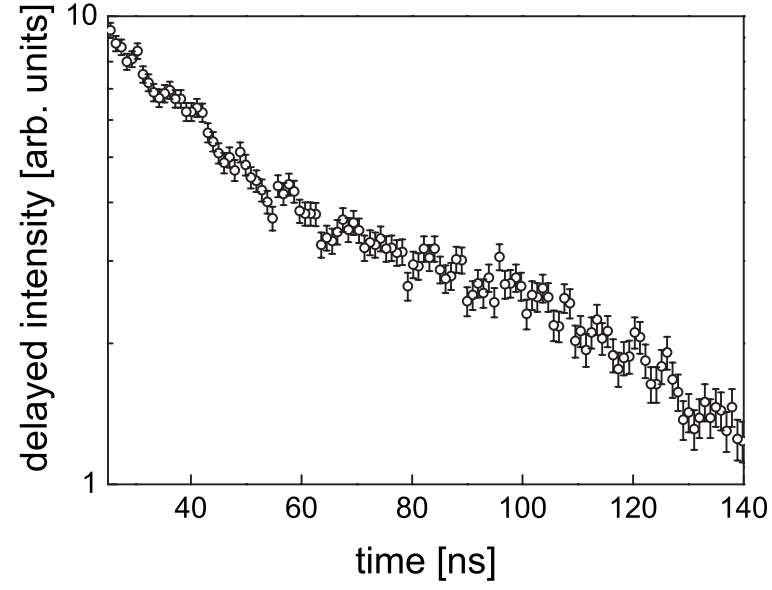

FIG. 10. Delayed intensity from nuclear resonant scattering at an Fe monolayer registered at room temperature after measurements at $770 \mathrm{~K}$.

conclude that diffusion in the Fe monolayer proceeds mainly via migration of vacancies. This yields for the diffusion energy $E_{d}$ (sum of $E_{f}$ and $E_{m}$ ) a value of $0.33(11) \mathrm{eV}$. Experimental values of $E_{d}$ spread from $0.5 \mathrm{eV}$ for the transition metal atoms $\mathrm{Ni}$ and $\mathrm{Pd}$ on $\mathrm{W}(110)$ up to $1.0 \mathrm{eV}$ for some other atoms (e.g, Re atoms) not including $\mathrm{Fe}^{2,3,5,6}$

Assuming a nearest neighbor jump diffusion mechanism the diffusion coefficient can be calculated according to $D$ $=l^{2} w c$ where $l$ is the length of the jump vector of the atom jump into a nearest neighbor site $l=(a / 2) \sqrt{3}, w$ is the vacancy jump frequency and $c$ is the vacancy concentration (both Table I). The data for $D$ are also given in Table I. The diffusion coefficient at $770 \mathrm{~K}$ is about twenty times larger than the diffusion coefficient of an Fe top atom (quasi a "self-adatom") on an Fe monolayer on W(110) at $820 \mathrm{~K}$ as derived from the data given by Reuter et al. ${ }^{22}$

\section{Remaining problems}

\section{Spectra of as-prepared monolayer measured at room temperature}

There is a slight beat in the room-temperature NRS spectrum (Fig. 3) which must be caused by a defect quadrupole interaction $Q_{D}$ felt by a small fraction of the $\mathrm{Fe}$ atoms in the monolayer. What may be the reason? The most appealing explanation is that vacancies have been quenched in, when after preparation at $770 \mathrm{~K}$ the monolayer was cooled down (fifteen minutes from 770 to $470 \mathrm{~K}$ and then more slowly down to room temperature). It is possible that a small fraction of the $11 \%$ vacancy concentration at $770 \mathrm{~K}$ (see Fig. 6 and Table I has remained in the monolayer down to room temperature.

\section{Structural changes at elevated temperatures}

The room-temperature NRS spectrum taken after $1.5 \mathrm{~h}$ measurements at $770 \mathrm{~K}$ (Fig. 10) was not completely identical to the original room-temperature spectrum from the asprepared monolayer: the beat from the high-temperature measurements has disappeared nearly completely, but there 
remains a somewhat faster decay which escapes reasonable fitting.

This implies that at long treatment at $770 \mathrm{~K}$ some irreversible minor change in the monolayer has happened. Searching for possible reasons for that structural modification after measuring the specimen at higher temperatures for longer time we find two alternatives. One is the formation of Fe dimers formed by the Fe atoms, which have left the afterwards vacant sites and remain immersed in the monolayer. Such dimers have been proposed by Spisak and Hafner ${ }^{41}$ who calculated that the dimers are rather resistant against breaking up which costs $0.7 \mathrm{eV}$ per atom. Another explanation may be based on the tendency of the pseudomorphically grown monolayer for relaxing through the formation of dislocations. Hints for such structural change may be found in the experimental work by Bethge et al. ${ }^{40}$ and by Przybylski ${ }^{42}$ who concluded that a dislocation network starts to appear for film thicknesses very slightly exceeding one monolayer. Keeping the specimens for longer time at elevated temperatures and afterwards cooling them down rather fast may have produced an incipient dislocation structure following the film's tendency to relax the pseudomorphic stress.

\section{Multiple vacancies}

The vacancy concentration $c$ resulting from the fit of the NRS spectra is about $4 \%$ at $570 \mathrm{~K}$ and increases up to about $11 \%$ at $770 \mathrm{~K}$. Caution is indicated regarding the value of fourteen percent estimated from the very strong beat in the spectrum taken at $870 \mathrm{~K}$, because of the just mentioned possible structural change having taken place at the long measuring at $870 \mathrm{~K}$. Simple Poisson statistics implies that at 570 $\mathrm{K}$ about $1.5 \%$ of all probe atoms face two vacancies at their four nearest neighbor sites, $4 \%$ at $670 \mathrm{~K}$ and $6 \%$ at $770 \mathrm{~K}$, the percentage of triple vacancies being negligible. Our present theory ${ }^{36}$ is unable to deal with the influence of double vacancies on the quadrupole interaction and we have to live with an uncertainty regarding the vacancy concentration determined by the fit, this uncertainty increasing with increasing temperature.

It is possible that the apparent change in the defect quadrupole interaction $Q_{D}$ (see Sec. IV) and the deviation of the data points from the fits at times longer than $120 \mathrm{~ns}$, both appearing with increasing temperature, are caused by the increasing fraction of double vacancies.

We stress that the merit of our study is to show that it is possible to observe defects, most probably vacancies and follow their diffusion by a surface scattering method. We note that the data quality is limited by the restricted beam time at present day's synchrotrons, i.e., why we have to put up with the fact that the results still contain considerable uncertainties.

\section{CONCLUSION AND PERSPECTIVES}

In conclusion we have gained the following results by applying nuclear resonant scattering of synchrotron radiation under grazing incidence at an Fe monolayer on W(110).

(i) With increasing temperature a distinct beat structure appears in the NRS spectra. It is ascribed to the formation of defects in the monolayer which leads to fluctuating quadrupole interactions acting on the nuclei and to a diffusionally accelerated decay of the intensity, both effects depending on the probability of a defect in the nuclei's neighborhood.

(ii) The NRS spectra are essentially reversible, i.e., the defect appearing when raising the temperature and disappearing again when lowering the temperature is a thermally activated defect. There is no indication for a significant contribution of foreign atoms, therefore we conclude that the defect is the vacancy.

(ii) The temperature dependence of the defect concentration is of Arrhenius type. We interpret the corresponding activation energy as the formation energy of the vacancy $E_{f}$ $=0.17(5) \mathrm{eV}$.

(iv) With increasing temperature the beats in the NRS spectra are increasingly smeared out and the decay of the intensity steepens. We tentatively interpret both effects as the consequence of the jumps of the vacancies within nanoseconds. The temperature dependence of the vacancy jump frequency is of Arrhenius type. The corresponding activation energy is the migration energy of the vacancy $E_{m}$ $=0.16(6) \mathrm{eV}$.

(v) For the activation energy for diffusion $E_{d}$ we estimate $0.33(11) \mathrm{eV}$, for the diffusion coefficient $0.15(13)$ $\times 10^{-12} \mathrm{~m}^{2} \mathrm{~s}^{-1}$ at $570 \mathrm{~K}$ rising to $3.4(1.2) \times 10^{-12} \mathrm{~m}^{2} \mathrm{~s}^{-1}$ at $870 \mathrm{~K}$.

The here presented NRS variant of Mössbauer spectroscopy, i.e., scattering of nuclear resonant synchrotron radiation in grazing incidence geometry, enables one to study simultaneously the structure and the dynamics on an atomic scale. Thanks to the high brilliance of synchrotron radiation from third generation synchrotrons this is even possible for monoatomic layers. Such a study was until recently completely inconceivable since the conventional scattering methods as neutron scattering ${ }^{16,17}$ and the resonance methods as, e.g., Mössbauer effect ${ }^{43-45}$ needed much more material than given in a monolayer in order to receive results in times which are feasible from work efficiency, beam time availability, and stability of device as well. STM on the other hand is not sufficiently sensitive for a vacancy due to problems with corrugation and FIM cannot directly determine the details of the vacancy dynamics. Besides both methods lack statistics and observe isolated individuals. They may, of course, be regarded as more "direct" for an observer who likes "direct vision," but we hold that a Fourier transformation is not less "true" than a computer image of the tunnel current.

We see the following perspectives of this investigation:

(i) Varying the penetration depth of the x-rays even buried layers and interfaces will become accessible using grazing incidence scattering of nuclear resonant X-rays. Please note that with surface sensitive microscopic methods (e.g., STM), atoms in layers below the surface cannot be seen.

(ii) In the present work the synchrotron beam was directed to the [110] direction of the monolayer. This angle can be varied and from the angular dependence of the NRS spectrum it will be possible to determine the vector of the single atom jump as has been successfully achieved in the bulk. ${ }^{34,35}$

(iii) It should be possible to determine the dynamics of adatoms in the same way as done here for vacancies. 
We finally repeat: the merit of our study is to show that it is possible to observe vacancies and follow their diffusion by a surface scattering method, even though the data quality is today still limited by the restricted beam time at present day's synchrotrons. Future measurements with highly improved brilliance of the synchrotron beam as will be available in the future at synchrotrons such as Petra III or the free-electron lasers will enable faster measurements, so that the statistics will be sufficient in order to gain more details on vacancy concentration, multiple defects, and diffusion.

\section{ACKNOWLEDGMENTS}

This work would not have been possible without the continuous cooperation with and advice by Józef Korecki and members of his group, in particular Tomasz Ślęzak and Nika Spiridis. We are grateful to Rudolf Rüffer for continuous support with beam time at ESRF, to Bogdan Sepiol for discussions, and to Fritz Gröstlinger and Michael Leitner for essential help. This work was financially supported by the Austrian FWF project No. P-20713-N20.

*gero.vog1@univie.ac.at

${ }^{1}$ H. Mehrer, Diffusion in Solids (Springer, New York, 2007).

${ }^{2}$ G. L. Kellogg, Jpn. J. Appl. Phys. 32, 1463 (1993).

${ }^{3}$ G. L. Kellogg, Surf. Sci. Rep. 21, 1 (1994).

${ }^{4}$ G. Antczak and G. Ehrlich, Surf. Sci. 62, 39 (2007).

${ }^{5}$ G. Ehrlich, Scanning Microsc. 4, 829 (1990).

${ }^{6}$ S. M. Oh, S. J. Koh, K. Kyuno, and G. Ehrlich, Phys. Rev. Lett. 88, 236102 (2002).

${ }^{7}$ D. Spisak and J. Hafner, Phys. Rev. B 70, 195426 (2004).

${ }^{8}$ R. Z. Huang, V. S. Stepanyuk, and J. Kirschner, J. Phys.: Condens. Matter 18, L217 (2006).

${ }^{9}$ A. J. Mayne, F. Rose, C. Bolis, and G. Dujardin, Surf. Sci. 486, 226 (2001).

${ }^{10}$ G. Ertl, Angew. Chem. Int. Ed. 47, 3524 (2008).

${ }^{11}$ S. Renisch, R. Schuster, J. Wintterlin, and G. Ertl, Phys. Rev. Lett. 82, 3839 (1999).

${ }^{12}$ M. Ø. Pedersen, L. Österlund, J. J. Mortensen, M. Mavrikakis, L. B. Hansen, I. Stensgaard, E. Lægsgaard, J. K. Nørskov, and F. Besenbacher, Phys. Rev. Lett. 84, 4898 (2000).

${ }^{13}$ S. Stankov, R. Rüffer, M. Sladecek, M. Rennhofer, B. Sepiol, G. Vogl, N. Spiridis, T. Ślęzak, and J. Korecki, Rev. Sci. Instrum. 79, 045108 (2008).

${ }^{14}$ S. Stankov, R. Röhlsberger, T. Ślęzak, M. Sladecek, B. Sepiol, G. Vogl, A. I. Chumakov, R. Rüffer, N. Spiridis, J. Łażewski et al., Phys. Rev. Lett. 99, 185501 (2007).

${ }^{15}$ A. Graham, Surf. Sci. Rep. 49, 115 (2003).

${ }^{16} \mathrm{G}$. Vogl and B. Sepiol, in Diffusion in Condensed Matter, edited by P. Heitjans and J. Kärger (Springer, New York, 2005), p. 65.

${ }^{17}$ T. Springer and R. E. Lechner, in Diffusion in Condensed Matter, edited by P. Heitjans and J. Kärger (Springer, New York, 2005), p. 93.

${ }^{18}$ U. Gradmann and G. Waller, Surf. Sci. 116, 539 (1982).

${ }^{19}$ H. J. Elmers, J. Hauschild, H. Fritzsche, G. Liu, U. Gradmann, and U. Köhler, Phys. Rev. Lett. 75, 2031 (1995).

${ }^{20}$ D. Sander, R. Skomski, C. Schmidthals, A. Enders, and J. Kirschner, Phys. Rev. Lett. 77, 2566 (1996).

${ }^{21}$ T.-U. Nahm and R. Gomer, Surf. Sci. 380, 52 (1997).

${ }^{22}$ D. Reuter, G. Gerth, and J. Kirschner, Phys. Rev. B 57, 2520 (1998).

${ }^{23}$ J. Kołaczkiewicz and E. Bauer, Surf. Sci. 450, 106 (2000).

${ }^{24}$ J. Berlowitz, J.-W. He, and D. W. Goodman, Surf. Sci. 231, 315

(1990).

${ }^{25}$ H. J. Elmers, G. Liu, and U. Gradmann, Phys. Rev. Lett. 63, 566 (1989).

${ }^{26}$ M. Przybylski and U. Gradmann, J. Appl. Phys. 63, 3652 (1988).

${ }^{27}$ M. Przybylski and U. Gradmann, Hyperfine Interact. 113, 135 (1998).

${ }^{28}$ H. J. Elmers, J. Hauschild, H. Höche, U. Gradmann, H. Bethge, D. Heuer, and U. Köhler, Phys. Rev. Lett. 73, 898 (1994).

${ }^{29}$ R. Röhlsberger, Nuclear Condensed Matter Physics with Synchrotron Radiation (Springer, New York, 2004).

${ }^{30}$ R. Rüffer and A. I. Chumakov, Hyperfine Interact. 97-98, 589 (1996).

${ }^{31}$ O. Hignette, C. Peffen, V. Alvaro, E. Chinchio, and A. K. Freund, Proc. SPIE 4501, 43 (2001).

${ }^{32}$ R. Röhlsberger, J. Bansmann, V. Senz, K. L. Jonas, A. Bettac, K. H. Meiwes-Broer, and O. Leupold, Phys. Rev. B 67, 245412 (2003).

${ }^{33}$ E. Partyka-Jankowska, B. Sepiol, M. Sladecek, D. Kmiec, J. Korecki, T. Slezak, M. Zajac, S. Stankov, R. Rüffer, and G. Vogl, Surf. Sci. 602, 1453 (2008).

${ }^{34}$ B. Sepiol, A. Meyer, G. Vogl, R. Rüffer, A. I. Chumakov, and A. Q. R. Baron, Phys. Rev. Lett. 76, 3220 (1996).

${ }^{35}$ B. Sepiol, A. Meyer, G. Vogl, H. Franz, and R. Rüffer, Phys. Rev. B 57, 10433 (1998).

${ }^{36}$ G. Vogl, M. Sladecek, and S. Dattagupta, Phys. Rev. Lett. 99, 155902 (2007)

${ }^{37}$ H. Grünsteudel, V. Rusanov, H. Winkler, W. Meyer-Klaucke, and A. X. Trautwein, Hyperfine Interact. 122, 345 (1999).

${ }^{38}$ G. V. Smirnov, Hyperfine Interact. 123-124, 31 (1999).

${ }^{39}$ M. Przybylski, J. Korecki, and U. Gradmann, Appl. Phys. A 52, 33 (1991).

${ }^{40}$ H. Bethge, D. Heuer, Ch Jensen, K. Reshöft, and U. Köhler, Surf. Sci. 331-333, 878 (1995).

${ }^{41}$ D. Spisak and J. Hafner, Surf. Sci. 584, 55 (2005).

${ }^{42}$ M. Przybylski, I. Kaufmann, and U. Gradmann, Phys. Rev. B 40, 8631 (1989).

${ }^{43}$ G. Vogl and B. Sepiol, Acta Metall. Mater. 42, 3175 (1994).

${ }^{44}$ R. Feldwisch, B. Sepiol, and G. Vogl, Acta Metall. Mater. 43, 2033 (1995).

${ }^{45}$ B. Sepiol and G. Vogl, Phys. Rev. Lett. 71, 731 (1993). 